EXTENDED REPORT

\title{
Expression of FGFR-2 and FGFR-3 in the normal human fetal orbit
}

\author{
S H Khan, J A Britto, R D Evans, K K Nischal
}

See end of article for authors' affiliations

....................

Correspondence to: Mr K K Nischal,

Department of Paediatric Ophthalmology, Great Ormond Street Hospital for Children, Great Ormond Street, London WC1N 3JH, UK; kkn@btinternet. com

Accepted for publication 1 August 2005
Br J Ophthalmol 2005;89:1643-1645. doi: 10.1136/bjo.2005.075978 (FGFR-2 and FGFR-3) in the normal human fetal orbit.

Methods: $6 \mu \mathrm{m}$ orbital slide sections were prepared from 12 week old human fetal material obtained within established ethical guidelines. Radioactive in situ hybridisation techniques were used to demonstrate the expression patterns of FGFR-2 and FGFR-3 within these sections. Only one foetus had appropriate orbital sections taken.

Results: FGFR-2 was expressed within the extraocular muscles (EOMs) and the optic nerve sheath and to a lesser degree within the orbital periosteal margins and the cranial sutures. FGFR-3 was expressed a lot within the periosteal margins and cranial sutures but not within either the EOMs or the optic nerve sheath. Conclusions: FGFR-2 and FGFR-3 are differentially expressed within different orbital components. FGFR-2 gene mutations may be responsible for craniosynostotic syndromes such as Crouzon, Pfeiffer, and Apert, while those in the FGFR-3 gene may cause isolated unicoronal synostosis. EOMs may be histologically abnormal in cases of Apert, Pfeiffer, and Crouzon syndromes but not isolated unicoronal synostosis. The pattern of expression of FGFR-2 in the normal human fetal orbit may explain some of the EOM histological findings seen in some cases of Apert, Pfeiffer, and Crouzon syndromes.
$M$ utations in the fibroblast growth factor receptor (FGFR) genes are known to result in craniosynostosis which is the abnormal premature fusion of one or more of the cranial sutures. ${ }^{1}$ The craniosynostoses may be syndromic or non-syndromic: mutations in FGFR-2 are most commonly responsible for the autosomal dominant syndromic craniosynostoses (such as Crouzon, Apert, and Pfeiffer), while the non-syndromic craniosynostoses may occur as a result of mutations in other genes including FGFR-3, which is known to be responsible for some cases of unicoronal synostosis. ${ }^{2}$

All the craniosynostotic syndromes have the common feature of craniosynostosis but vary in their combination of other anomalies of bone differentiation, which mainly affect their hands and feet ${ }^{3}$; for example, in Apert syndrome there is typically syndactyly while in Pfeiffer syndrome there is an anomalous broad thumb.

The position of the rectus muscles in patients with craniosynostoses is often exocyclorotated. ${ }^{4}$ This is thought to be due to a mechanical exocyclorotation of the orbits secondary to premature cranial synostosis ${ }^{4}$ and may be seen in non-syndromic and syndromic cases.

However, anomalies of extraocular muscle (EOM) structure have only been described in the syndromic craniosynostoses and not in the non-syndromic unicoronal, bicoronal, sagittal, or metopic craniosynostoses. ${ }^{5-8}$

Captuo and Lingua ${ }^{5}$ reported abnormal bifid medial rectus and anomalous insertions of both horizontal rectus muscles in a patient with Crouzon syndrome. Absence and/or hypoplasia of EOMs has also been shown to occur with increased frequency in Crouzon syndrome. ${ }^{6}$ Margolis et al ${ }^{7}$ described structural alterations in extraocular muscle fibres as seen by light and electron microscopy in a patient with Apert syndrome, while Greenberg and Pollard ${ }^{8}$ have described both muscle hypoplasia and also absence of multiple EOMs in patients with both Pfeiffer and Apert syndromes. Findings of a fibrous band in lieu of rectus muscle are not a surprise when operating on these patients and have necessitated novel approaches to the treatment of their eye movement disorders. ${ }^{9}$

We studied the expression patterns of FGFR-2 and FGFR-3 in a normal human fetal orbit in an effort to explain the above clinical findings.

\section{METHODS}

We looked at the expression of FGFR-2 and FGFR-3 in a 12 week old human fetal orbit as per methodology previously reported..$^{10}$

Human fetuses of 12 weeks of age were collected under the aegis of the Developmental Biology Tissue Resource maintained at the Institute of Child Health and University College, London. The fetuses were obtained within established ethical guidelines, from the social termination of pregnancy by RU486 or surgical methods. None was terminated for reasons of fetal abnormality but no formal karyotyping was performed. The fetal age was determined by the magnified assessment of external morphology. All fetuses were fixed in $4 \%$ paraformaldehyde, in phosphate buffered saline at $4^{\circ} \mathrm{C}$ for 2-5 days, then rinsed in phosphate buffered saline, and passed through increasing concentrations of phosphate buffered saline/ethanol solutions until storage at $4^{\circ} \mathrm{C}$ in $70 \%$ ethanol. The specimens were then dehydrated through an alcohol series, cleared in Histoclear (RA Lamb, East Sussex, UK), embedded in paraffin wax, and serially sectioned at $6 \mu \mathrm{m}$ onto TESPA stubbed slides. Only one fetus had the appropriate sections taken through the orbits.

Slides were examined using an Olympus $\mathrm{BH} 2$ microscope, and images were captured electronically using a Kontron ProgRes3012 digital camera, version 2 of the associated software, and stored as Adobe Photoshop version 5.0 files.

Abbreviations: EOMs, extraocular muscles; FGFR, fibroblast growth factor receptors; ICP, intracranial pressure 


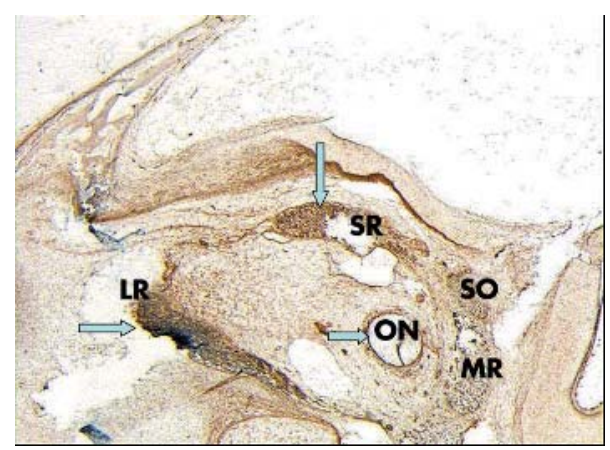

Figure 1 Expression of FGFR2-dark brown indicates positive stain (arrows)-predominantly within muscles and optic nerve sheath. LR, lateral rectus; SR, superior rectus; $M R$, medial rectus; $S O$, superior oblique; ON, optic nerve.

\section{RESULTS}

FGFR-2 expression was positively demonstrated within the extraocular muscles and also within the optic nerve sheath. It was also demonstrated to a lesser degree within the periosteal margins of the orbit and within the cranial sutures (see fig 1).

FGFR-3 was not demonstrated within either the extraocular muscles or the optic nerve sheath, but was more positively expressed than FGFR-2 within the periosteal margins and cranial sutures (see fig 2).

\section{DISCUSSION}

Fibroblast growth factors (FGF) are a family of structurally similar mitogenic factors that exert their key effects within the body via their roles in the growth and differentiation of various tissues of mesenchymal and neuroectodermal origin. ${ }^{2}$ They are also important for chemotaxis, angiogenesis, and cell apoptosis. The key members of this family are acidic FGF (FGF-1) and basic FGF (FGF-2), which exert their effects by interacting with membrane spanning tyrosine-kinase receptors. There are four types of high affinity receptors, simply known as FGFR 1-4. Binding by fibroblast growth factors to these receptors causes receptor transphorylation and activation of their kinase domains.

FGFR-2 and FGFR 3 staining in a normal human orbit has not previously been reported to the best of our knowledge. Although the findings are in only one fetus it is nevertheless valuable information and perhaps a first step to phenotypegenotype correlation.

Apert, Crouzon, and Jackson-Weiss syndromes result from FGFR-2 gene mutations. When Pfeiffer syndrome is the result of an FGFR-2 mutation, it is not possible to distinguish between it, Crouzon or Jackson-Weiss even at a molecular level, while Apert syndrome can be seen to be an allelic variation of these syndromes. ${ }^{11}$ Pfeiffer syndrome may also result from mutations in either FGFR-1 (chromosome 8) or FGFR-2 (chromosome 10).

The FGFR-3 gene is found on chromosome 4 and mutations here may result in Muenke's syndrome ${ }^{2}$ which is also known as non-syndromic coronal synostosis, involving one or both coronal sutures. Owing to the incomplete penetrance of this anomaly, it is suggested that all cases of coronal synstosis should be assessed for this FGFR3 mutation.

Our results demonstrate positive expression of FGFR-2 but not FGFR-3 in the EOMs. This may explain the abnormal EOM structure described in the literature to be found in patients with syndromic craniosynostoses such as Apert, Pfeiffer, and Crouzon. FGFR-2 mutation is known to cause a loss of regulation/gain of function defect (ligand independent

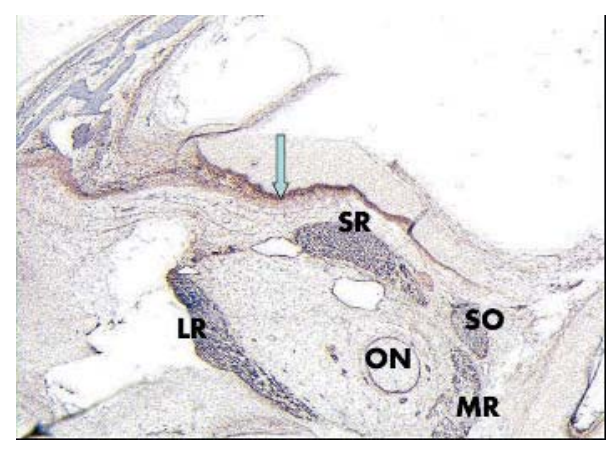

Figure 2 Expression of FGFR3 - dark brown indicates positive stain (arrows)-periosteal margins and sutures but not in muscle or optic nerve sheath. LR, lateral rectus; $S R$, superior rectus; $M R$, medial rectus; $\mathrm{SO}$, superior oblique; $\mathrm{ON}$, optic nerve.

activation), resulting in uncontrolled growth and differentiation of its target tissues. ${ }^{2}$ This in turn may also lead to the production of the excessive fibrous tissue seen in rectus muscle analysis from such patients. ${ }^{11}$ Anecdotally, these muscles feel gristly and inelastic when manipulated during operation.

Light microscopy of muscles in Apert syndrome shows cells in varying stages of degeneration scattered among a majority of normal looking muscle fibres; other muscle fibres are described as enlarged and hyalinised. ${ }^{7}$ Electron microscopy further shows these hyalinised fibres to have a loss of myofibrillar organisation and to contain large clusters of mitochondria, which in themselves display vacuolation, disruption, and fragmentation of cristae. A variety of nuclear abnormalities are also described, with subsarcolemmal inclusions being seen frequently.

It is interesting to note that similar mitochondrial abnormalities have been described in both myopathic and neurogenic diseases where altered movements are a feature, while granular inclusions similar to the subsarcolemmal inclusions have previously been described in myotonic dystrophy and myasthenic muscles.

The fact that FGFR-3 mutation related conditions such as Muenke's have not been described as having structurally abnormal or absent EOMs is consistent with our finding of a lack of expression of FGFR-3 in the EOMs.

Our results also demonstrate positive expression of FGFR-2 but not FGFR-3 in the optic nerve sheath. Absence of optic disc swelling in the presence of confirmed raised intracranial pressure has been well described in the syndromic craniosynostoses and may be seen in up to $50 \%$ of cases with confirmed raised intracranial pressure (ICP). ${ }^{12} 13$

Although the absence of disc swelling in the presence of raised ICP in these patients may be attributed to the presence of optic atrophy in some cases, this is not likely to be true in every case. The Schmidt-Manz transport theory describes optic disc swelling in raised ICP to occur as a result of cerebrospinal fluid being forced into intravaginal spaces of the optic nerve sheath within the lamina cribrosa, resulting in oedema and incarceration of the optic nerve head..$^{14}$ In cases where there is a lack of optic disc swelling, it suggests that there must be an obstruction to the communication between the cranial cavity and the subvaginal spaces of the optic nerve sheath to prevent development of the oedema. ${ }^{15}$ Based on our findings, we speculate that the lack of optic disc swelling in the presence of raised ICP in cases of syndromic craniosynostosis may be the result of abnormal fibrous tissue in the optic nerve sheath and/or the lamina cribrosa. Unfortunately, at present no optic nerve sheath histology is available in a patient with FGFR2 mutation related 
syndromic craniosynostosis to be able to determine whether fibrous changes do occur in the sheath and lamina cribrosa.

Therefore, whether the expression of FGFR-2 in optic nerve sheath might explain the high incidence of absence of papilloedema seen in syndromic craniosynostoses despite documented raised ICP, remains purely speculative at present.

In summary, we have shown expression patterns of FGFR2 in the normal human fetal orbit, contrasting with expression patterns of FGFR-3, which provide a reasonable platform for explaining some of the clinical and histopathological features of EOMs in patients with syndromic craniosynostosis.

\section{Authors' affiliations}

S H Khan, K K Nischal, Department of Paediatric Ophthalmology, Great Ormond Street Hospital for Children, Great Ormond Street, London WCIN 3JH, UK

S H Khan, K K Nischal, Visual Sciences Unit, Institute of Child Health, 30 Guilford Street, London WC1N 1EH, UK

J A Britto, R D Evans, K K Nischal, Craniofacial Unit, Great Ormond Street Hospital for Children, Great Ormond Street, London WCIN 3JH, UK

Competing interests: none declared

\section{REFERENCES}

1 Khan SH, Nischal KK, Dean F, et al. Visual outcomes and amblyogenic risk factors in the syndromic craniosynostoses - a review of 141 cases. Br J Ophthalmol 2003;87:999-1003.
2 Vajo Z, Francomano CA, Wilkin D. The molecular and genetic basis of fibroblast growth factor receptor 3 disorders: The achondroplasia family of skeletal dysplasias, Muenke craniosynostosis, and Crouzon syndrome with acanthosis nigricans. Endocrine Rev 2000;21:23-39.

3 De Moerlooze L, Dickson C. Skeletal disorders associated with fibroblast growth factor receptor mutations. Curr Opin Genet Develop 1997;7:378-85.

4 Cheng $\mathbf{H}$, et al. Dissociated eye movements in craniosynostosis: a hypothesis revived. Br J Ophthalmol 1993;77:563-8.

5 Captuo A, Lingua R. Abberrant muscle insertions in Crouzon's disease. J Pediatr Ophthalmol Strabismus 1980;17:239-41.

6 Diamond GR, Katowitz JA, Whittaker LA, et al. Variations in extraocular muscle number and structure in craniofacial dysostosis. Am J Ophthalmol 1980;90:416-18.

7 Margolis S, Pachter B, Breinin G. Structural alterations of extraocular muscle associated with Apert's syndrome. Br J Ophthalmol 1977;61:683-9.

8 Greenberg M, Pollard Z. Absence of multiple extraocular muscles in craniosynostosis. J AAPOS 1998;2:307-9.

9 Rattigan S, Nischal KK. Foster-type modification of the Knapp procedure for anomalous superior rectus muscles in syndromic craniosynostoses. J AAPOS 2003;7:279-82.

10 Britto JA, Chan JC, Evans RD, et al. Differential expression of fibroblast growth factor receptors in human digital development suggests common pathogenesis in complex acrosyndactyly and craniosynostosis. Plast Reconstr Surg 2001; 107:1331-8.

11 Steinberger D, Reinhartz T, Unsold R, et al. FGFR2 mutation in clinically nonclassifiable autosomal dominant craniosynostosis with pronounced phenotypic variation. Am J Med Genet 1996;66:81-6.

12 Tuite GF, Chong WK, Evanson J, et al. The effectiveness of papilledema as an indicator of raised intracranial pressure in children with craniosynostosis. Neurosurgery 1996;38:272-8.

13 Liasis A, Thompson DA, Hayward R, et al. Sustained raised intracranial pressure implicated only by pattern reversal visual evoked potentials after cranial vault expansion surgery. Pediatr Neurosurg 2003;39:75-80.

14 Somani S, Mackeen LD, Morad Y, et al. Assessment of extraocular muscles position and anatomy by 3-dimensional ultrasonography: a trial in craniosynostosis patients. J AAPOS 2003;7:54-9.

15 Hayreh SS. Pathogenesis of oedema of the optic disc-the mechanical theory. Thesis 1965;Chapter 2:16-21. 